\title{
Therapeutic effects of mesenchymal stem cell- derived microvesicles on pulmonary arterial hypertension in rats
}

\author{
Jian-ying $\mathrm{CHEN}^{1, \#, *}$, Ran $\mathrm{AN}^{1, \#}$, Zhen-jun LIU ${ }^{1, \#}$, Jin-ju WANG ${ }^{2}$, Shu-zhen $\mathrm{CHEN}^{2}$, Mian-ming HONG ${ }^{1}$, Jing-hu LIU ${ }^{1}$, \\ Meng-yuan $\mathrm{XIAO}^{1}$, Yan-fang $\mathrm{CHEN}^{2, *}$ \\ ${ }^{1}$ Department of Cardiology, the Affiliated Hospital of Guangdong Medical College, Zhanjiang 524001, China; ${ }^{2}$ Department of Pharma- \\ cology and Toxicology, Boonshoft School of Medicine, Wright State University, Dayton, OH 45435, USA
}

\begin{abstract}
Aim: Microvesicles (MVs) are nanoscale membrane fragments released from virtually all cell types upon activation or apoptosis, and may contribute to the beneficial effects of stem cell therapy. In this study, we investigated the therapeutic effects of mesenchymal stem cell (MSC) derived MVs (MSC-MVs) on pulmonary artery hypertension (PAH) in rats.

Methods: MSC-MVs were isolated from rat bone marrow MSCs that were cultured in a serum-free conditioned medium. Transmission electron microscopy (TEM), flow cytometry and nanoparticle tracking analysis (NTA) were used to characterize the MVs. Adult SD rats were injected with monocrotaline $(50 \mathrm{mg} / \mathrm{kg}, \mathrm{sc})$ to induce PAH. Three weeks later, the rats were intravenously injected with MSCs, MSC-MVs or saline for 2 weeks. At the end of treatments, the hemodynamic parameters and pathological right ventricular and pulmonary arterial remodeling were analyzed in each group.

Results: The MSC-MVs showed general morphologic characteristics of MVs and expressed annexin V and CD29 markers under TEM, and their size ranged from 40 to $300 \mathrm{~nm}$. Intravenous injection of MSC-MVs or MSCs significantly ameliorated the mean pulmonary artery pressure (mPAP) and mean right ventricle pressure (mRVP) in PAH rats. Furthermore, intravenous injection of MSC-MVs or MSCs significantly decreased the right ventricle (RV) hypertrophy and pulmonary arteriole area index (AI) and thickness index (TI) in PAH rats. Conclusion: Intravenous injection of MSC-MVs or MSCs produces similar beneficial effects for treating PAH, and our results provide a basis for cell-free approach in stem cell therapy.
\end{abstract}

Keywords: stem cell therapy; bone marrow mesenchymal stem cell; microvesicle; pulmonary artery hypertension; chronic heart disease

Acta Pharmacologica Sinica (2014) 35: 1121-1128; doi: 10.1038/aps.2014.61; published online 4 Aug 2014

\section{Introduction}

Pulmonary artery hypertension (PAH) is a progressive chronic disease with a high mortality rate. $\mathrm{PAH}$ is characterized by increased pulmonary arterial pressure, which results in the right ventricle (RV) hypertrophy and ultimately RV failure ${ }^{[1]}$. Current treatments for PAH include endothelin receptor blockers, prostanoids and calcium channel blockers ${ }^{[2]}$. Recently, cell-based therapy has emerged as a promising approach for $\mathrm{PAH}$. Intravenous injection of bone marrow-derived progenitors $^{[3]}$ or mesenchymal stem cells (MSCs) ${ }^{[4]}$ has been shown to inhibit arteriole thickening and to improve the RV function in a rat PAH model. In addition, intratracheal application of

\footnotetext{
"These authors contributed equally to this work.

* To whom correspondence should be addressed.

E-mail yanfang.chen@wright.edu (Yan-fang CHEN); jychen271@126.com (Jian-ying CHEN)

Received 2014-01-02 Accepted 2014-05-26
}

MSCs has been shown to restore the pulmonary response to acetylcholine and decrease pulmonary arterial pressure, with no detectable immunolabeled MSCs in the walls of pulmonary vessels $^{[5]}$. This study provides good evidence supporting the paracrine effect of stem cell-based therapy. However, the detailed mechanism needs in-depth exploration.

Microvesicles (MVs) are nanoscale membrane fragments that can be released from virtually all cell types upon activation or apoptosis. MVs carry cargo, including specific proteins, mRNAs and microRNAs of their parent cells, and participate in cell-to-cell communication ${ }^{[6]}$. The therapeutic effects of MVs have been actively investigated in animal models of various diseases. For example, MSC-MV transplantation could protect kidneys from ischemia/reperfusion-induced injury and chronic renal damage by stimulating proliferation and inhibiting the apoptosis of tubular epithelial cells ${ }^{[7-9]}$. Intravenous administration of MSC-MVs has been shown to improve neurogenesis and angiogenesis in an ischemic stroke model ${ }^{[10]}$. 
We found that endothelial progenitor cell-MVs could protect endothelial cells against hypoxia-induced injury in in vitro culture systems ${ }^{[11]}$. Furthermore, a recent study indicated that MSC-MVs acquire the function of MSCs and can inhibit vascular remodeling and hypoxic pulmonary hypertension in a hypoxia-induced pulmonary hypertension mouse model ${ }^{[12]}$, indicating a novel application for stem cell-based therapy.

In this study, we aimed to investigate the therapeutic effects of MSC-MVs for treating PAH in a monocrotaline (MCT)induced PAH rat model.

\section{Materials and methods}

\section{Animals and study design}

Sprague Dawley (SD) rats (2-8 weeks old) were purchased from the Animal Experimental Center of Guangdong Province (Guangzhou, China) and housed in the animal care facility at the Affiliated Hospital of Guangdong Medical College. All rats were kept under standard temperature, humidity, and time light conditions and fed standard chow and water ad libitum. All experimental protocols were approved by the Institutional Animal Care and Use Committee of the Affiliated Hospital of Guangdong Medical College.

Monocrotaline (MCT) was diluted in saline before injection as previously described ${ }^{[13]}$. On d $1, \mathrm{SD}$ rats (6-8 weeks old) received a single subcutaneous injection of MCT $(50 \mathrm{mg} / \mathrm{kg}$ body weight) to induce PAH $(n=21)$, or saline alone to serve as a control group $(n=7)$. After 3 weeks, the rats injected with MCT were randomly assigned to 3 groups ( $n=7 /$ group): vehicle (saline), MSC (treated with $1 \times 10^{6}$ cells $/ 100 \mu \mathrm{L}$ saline, once), and MSC-MV (treated with $30 \mu \mathrm{g} / 100 \mu \mathrm{L}$ saline, once/ $2 \mathrm{~d}$ for 2 weeks). At the end of the experiment, the hemodynamic parameters and pathological right ventricular (RV) and pulmonary arterial remodeling were analyzed in each group.

\section{Isolation, culture, and characterization of MSCs}

MSCs were isolated from the bone marrow of rats as we previously described ${ }^{[7]}$. Briefly, after the rat was sacrificed, the femurs and tibias were removed and cleaned. Cells were collected and dispensed in a $15-\mathrm{mL}$ centrifuge tube, centrifuged at $200 \times \mathrm{g}$ for $6 \mathrm{~min}$, and resuspended with MSC culture medium, which was composed of a-minimal essential medium (a-MEM, Gibco, NY, USA) supplemented with 10\% FBS and $1 \%$ antibiotic-antimycotic solution. Cells were plated in a culture dish and cultured in a humidified 5\% $\mathrm{CO}_{2} / 95 \%$ air incubator.

Flow cytometry analysis was performed to identify cultured cells $^{[7]}$. In brief, cells were detached using trypsin-EDTA, centrifuged and incubated with PE-conjugated antibodies in a final volume of $100 \mathrm{~mL}$ for $30 \mathrm{~min}$ at $4{ }^{\circ} \mathrm{C}$ in the dark. Antibodies (PE-conjugated anti-CD29, PE-conjugated anti-CD45, PEconjugated anti-CD31, and PE-conjugated mouse IgG1 isotype controls) were purchased from BD Biosciences (San Jose, CA, USA). The concentrations of antibodies were applied according to the manufacturer's instructions. Labeled cells were washed and analyzed using flow cytometric analysis (Coulter Epics XL_MCL flow cytometer; Beckman Coulter). A total of at least 10000 events were collected and analyzed.

The multipotent differentiation ability of cultured cells was evaluated as previously reported ${ }^{[4]}$. For adipogenic differentiation, $5 \times 10^{3}$ cells/well were seeded in a 12-well culture plate and cultured with adipogenic differentiation medium, which was composed of DMEM (Gibco, NY, USA) supplemented with 10\% FBS, $10 \mu \mathrm{g} / \mathrm{mL}$ insulin (Sigma, MO, USA), $1 \mu \mathrm{mol} / \mathrm{L}$ dexamethasone (Sigma, MO, USA), $200 \mu \mathrm{mol} / \mathrm{L}$ indomethacin (Sigma, MO, USA), $0.5 \mathrm{mmol} / \mathrm{L}$ IBMX (Sigma, MO, USA) and $1 \%$ antibiotic-antimycotic solution (Gibco, NY, USA) for 3 weeks. The medium was changed every $3-4 \mathrm{~d}$. Lipid accumulation was assessed by oil red $\mathrm{O}$ staining of the cultures (15 $\mathrm{mg}$ of oil red $\mathrm{O} / \mathrm{mL}$ of $60 \%$ isopropanol). For osteogenic differentiation, $5 \times 10^{3}$ cells/well were seeded in a 12-well culture plate and cultured with osteogenic differentiation medium, DMEM supplemented with $10 \mathrm{mmol} / \mathrm{L} \beta$-glycerophosphate, $50 \mu \mathrm{g} / \mathrm{mL}$ ascorbic acid, and $100 \mathrm{nmol} / \mathrm{L}$ dexamethasone (all were purchased from Sigma) for 3 weeks. The medium was changed every 3-4 d. Calcium deposits were visualized by staining with $2 \%$ alizarine red $\mathrm{S}$ in $0.5 \% \mathrm{NH}_{4} \mathrm{OH}(\mathrm{pH}=5.5)$. All experiments were conducted in triplicate.

\section{Collection and characterization of MSC-MVs}

MSC-MVs were obtained from MSC conditioned culture medium according to a previously described protocol with slight modifications ${ }^{[7]}$. Briefly, MSCs were cultured in serumfree a-MEM for $24 \mathrm{~h}$. Then, the cell culture medium was collected, centrifuged at $2000 \times g$ for $20 \mathrm{~min}$ to remove cells and debris. The supernatants were ultracentrifuged at $100000 \times g$ for $1 \mathrm{~h}$ to pellet the MVs. The protein concentration of the MV preparations was quantified by the Bradford method (Bio-Rad, Hercules, USA).

MSC-MVs were characterized using flow cytometry, transmission electron microscopy (TEM) and nanoparticle tracking analysis (NTA). For flow cytometry analysis, MSC-MVs were resuspended and incubated for $30 \mathrm{~min}$ at $4^{\circ} \mathrm{C}$ in the dark with PE-conjugated CD29 and FITC-conjugated annexin V. An isotype-matched (IgG) nonspecific antibody served as a negative control. All antibodies were purchased from BD Biosciences (San Jose, CA, USA). After incubation, labeled cells were washed with PBS three times and resuspended with $70 \mu \mathrm{L}$ of PBS for flow cytometric analysis (Coulter Epics XL_MCL flow cytometer; Beckman Coulter). For TEM analysis, MVs were resuspended in PBS and loaded onto mesh nickel Formvar carbon coated grids (Electron Microscopy Science, Hatfield, PA, USA). Excess fluid was removed with filter paper, and the samples were stained with $1 \%$ uranyl acetate for $30 \mathrm{~s}$ and then washed, dried and stained with $2 \%$ lead citrate for another $30 \mathrm{~s}$. The grids were examined under a transmission electron microscope. All experiments were conducted in triplicate.

The size of the collected MSC-MVs was analyzed using a NanoSight NS300 with a 405-nm laser (NanoSight Ltd, Amesbury, UK) according to the previously described method ${ }^{[14]}$. Briefly, approximately $0.3 \mathrm{~mL}$ of MSC-MVs resuspended in PBS were loaded into the chamber of an NS300 unit. A 405$\mathrm{nm}$ laser beam was used to highlight the particles, which act 
as point scatters. Three 60 -second videos were taken. A minimum of 200 completed particle tracks in each video was analyzed by NTA2.3 software. The velocity of particle movement was used to calculate particle size using the two dimensional Stokes-Einstein equation.

\section{Tail vein injection of MSC-MVs and MSCs}

After 3 weeks of MCT injection, rats received MSC or MSC-MV administration via the tail vein as we previously described ${ }^{[15]}$. Briefly, MSCs $\left(1 \times 10^{6} / 100 \mu \mathrm{L}\right)$ were slowly injected via the tail vein once $^{[4]}$. MSC-MVs $(30 \mu \mathrm{g} / 100 \mu \mathrm{L})$ were injected on alternate days for 2 weeks according to a previous report ${ }^{[7]}$. In the control and vehicle groups, rats received $100 \mu \mathrm{L}$ of saline via tail vein injection.

\section{Measurement of pulmonary hemodynamics}

Pulmonary hemodynamics were measured as previous described $^{[16]}$. Briefly, 2 weeks after MCT administration, the experimental rats were anesthetized. Then, a polyethylene catheter filled with $2 \%$ sodium heparin was inserted into the RV through the jugular vein and the carotid artery for hemodynamic measurements. The RV systolic pressure and systemic blood pressure were measured by a pressureconductance catheter (model FT212, SciSense, Canada) coupled with a polygraph system (Medlab, Nanjing, China).

\section{Assessment of right heart hypertrophy}

After systemic arterial and RV pressures had been recorded, the rats were euthanized, and the lungs and hearts were isolated. The RV, left ventricle (LV) and ventricular septum (VS) were collected, dried with filter paper and weighed. RV hypertrophy was expressed as RV/(LV+VS).

\section{Evaluation of pulmonary arteriole remodeling}

After aortic perfusion with $4 \%$ PFA, the lungs were excised and fixed in $4 \%$ PFA overnight at $4{ }^{\circ} \mathrm{C}$ followed by paraffin embedding. Then, lung sections $(4 \mu \mathrm{m})$ were prepared and stained with hematoxylin and eosin (H\&E). Sections were examined under a light microscope (Olympus, Japan). The pulmonary arteriole wall thickness index (TI) and area index (AI) were calculated by the following formulas: TI=(external diameter-internal diameter)/external diameter $\times 100 \%$ and $\mathrm{AI}=($ cross section area-lumen area)/cross section area $\times 100 \%$. The mean TI and AI were calculated from 5 random cross sections of each pulmonary arteriole.

\section{Statistical analysis}

Data were expressed as the mean \pm SEM. Comparisons of two groups were performed by Student's $t$-test. Multiple comparisons were performed by one- or two-way ANOVA. SPSS software version 17.0 was used. For all tests, $P<0.05$ was considered to be statistically significant.

\section{Results}

MSCs were positive for specific stromal cell markers and could be differentiated into adipocytes and osteocytes

The cultured cells displayed a fibroblast-like morphology (Figure $1 \mathrm{~A})$. As determined by flow cytometric analysis, cultured
A

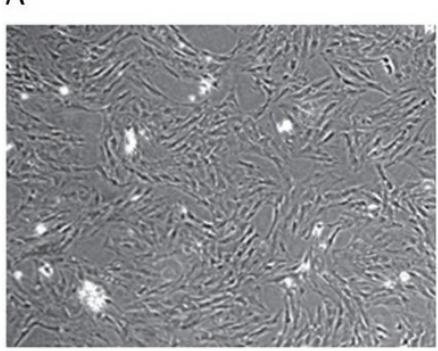

D



B



C

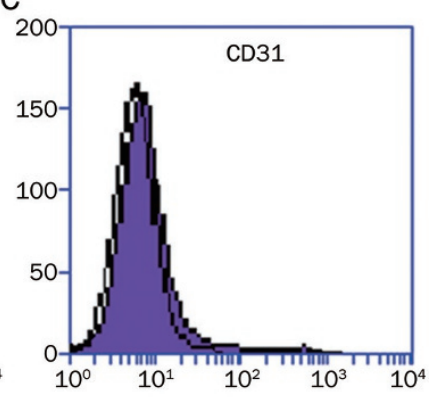

$\mathrm{F}$



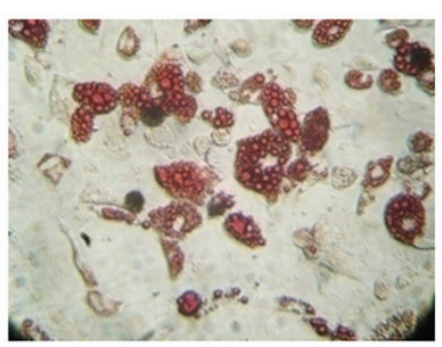

Figure 1. MSC culture and characterization. (A) A representative plot shows the fibroblast-like morphology of cultured MSCs. (B-D) Representative flow cytometry plots show the expression of CD29, but not CD31 and CD45, in cultured MSCs. Left curves, isotype control; Right curves, antibodies. (E) Osteogenic differentiation capacity of MSCs confirmed by alizarin red S staining. (F) Adipogenic differentiation capacity of MSCs determined using oil red $\mathrm{O}$ staining. Magnification in $\mathrm{A}, \mathrm{E}$, and $\mathrm{F}: \times 10$. 
cells positively expressed the stromal stem cell marker CD29 (Figure 1B) and negatively stained with hematopoietic lineage markers CD31 and CD45 (Figure 1C and 1D). The differentiation capacity of MSCs was revealed by the successful differentiation of MSCs into osteocytes, with calcium deposition within the cells (Figure 1E), and adipocytes, confirmed by the accumulation of lipid droplets in the vacuoles (Figure $1 F$ ).

\section{Characterization of MSC-MVs}

MSC-MVs were generated from MSCs exposed to serumfree medium as described in the Materials and methods. The flow cytometry results revealed that the majority of MSC-MVs had externalized phosphatidylserine, which was detected by annexin V staining, and expressed the MSC specific marker CD29 (Figure 2B). In addition, MSC-MVs displayed a spherical shape under TEM, as indicated by arrows (Figure 2C). Figure 2D showed that the size of MSC-MVs mainly (>95\%) ranged from 40 to $300 \mathrm{~nm}$ with a peak size of approximately $134 \mathrm{~nm}$, as analyzed by NTA. These data confirm the proper isolation of MSC-MVs in our study.

MSC-MVs and MSCs significantly attenuated mPAP and mRVP in $\mathrm{PAH}$ rats

At week 5 after MCT injection, pulmonary hemodynamics were measured in all rats. Representative pressure curves of mPAP and mRVP are shown in Figure 3A1-3A4. The PAH model was produced successfully as revealed by a higher mPAP in the vehicle group than in the control group $(P<0.01$; $n=7$ /group). Also, mPAP was attenuated in the MSC-MV and
MSC groups compared to the vehicle group but was higher than that in the control group $(P<0.01 ; n=7 /$ group) (Figure $3 \mathrm{~B})$. There was no difference in MPAP between the MSC and MSC-MV groups ( $P>0.05 ; n=7 /$ group). Similarly, mRVP was lower in the MSC-MV and MSC groups than that in the vehicle group but was higher than that in the control group $(P<0.01$; $n=7$ /group). Also, there was no significant difference in these variables between the MSC and MSC-MV groups $(P>0.05$; $n=7$ /group).

\section{MSC-MV treatment significantly decreased RV hypertrophy in} $\mathrm{PAH}$ rats

$\mathrm{RV}$ hypertrophy was quantified by the weight ratio of RV/ $(\mathrm{LV}+\mathrm{VS})$. The weight ratio of $\mathrm{RV} /(\mathrm{LV}+\mathrm{VS})$ in the MSC-MV group was significantly lower than that in the vehicle group but was higher than that in the control group $(P<0.01 ; n=7 /$ group) (Figure 4A). In the MSC group, the average ratio of $\mathrm{RV} /(\mathrm{LV}+\mathrm{VS})$ was not different from that in MSC-MV group $(P>0.05 ; n=7 /$ group $)$.

MSC-MV administration reduced pulmonary arteriole remodeling H\&E staining demonstrated the wall thickening and luminal stenosis in the four different groups (Figure 4B). The pulmonary arteriole thickness index (TI) and area index (AI) were significantly decreased in the MSC-MV and MSC groups compared to those in the vehicle group and were higher than those in the control group $(P<0.01 ; n=7$ /group; Figure $4 \mathrm{C}$ and $4 \mathrm{D})$. Again, there were no significant differences in these variables between the MSC and MSC-MV groups ( $P>0.05 ; n=7$ /group).
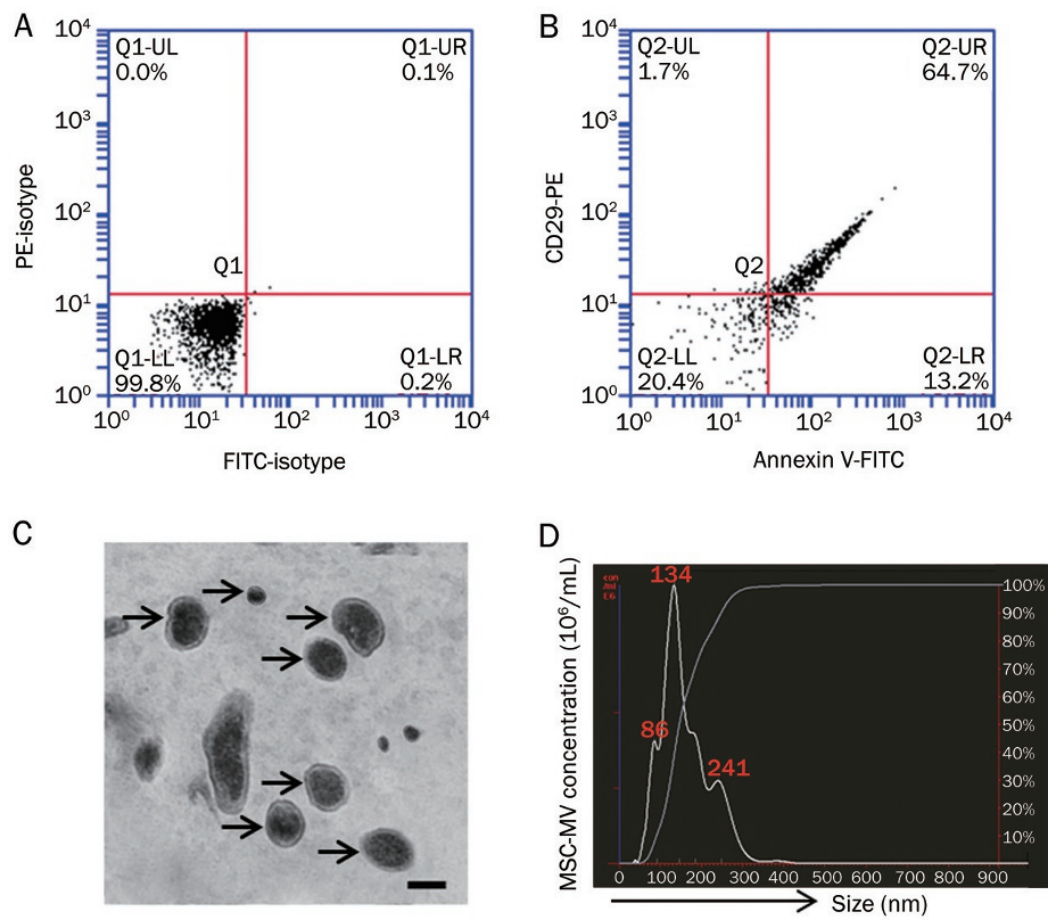

Figure 2. MSC-MVs characterized by flow cytometry, transmission electron microscopy and NTA. (A) Isotype controls for MSC-MVs. (B) Annexin V and MSC-specific marker CD29 on MSC-MVs. (C) MSC-MVs viewed under transmission electron microscope. Arrows indicate MSC-MVs. Scale bar: 0.2 um. (D) Representative graph shows the size distribution of MSC-MVs as determined by NTA. 

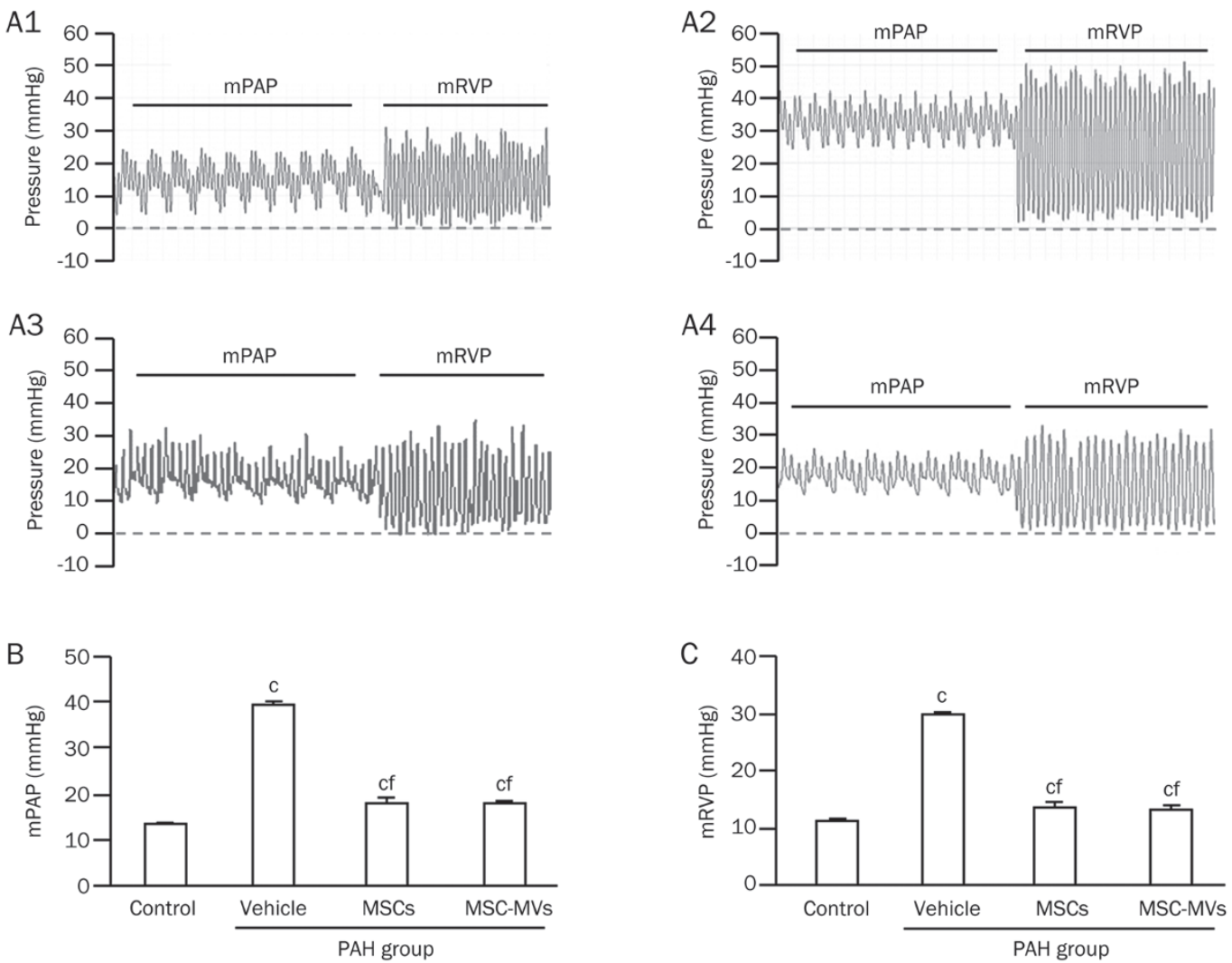

Figure 3. MSC-MVs and MSCs alleviate mPAP and mRVP in PAH rats. (A1-A4) Representative traces show the mPAP and mRVP in control, vehicle, MSC, and MSC-MV groups. (B and C) Summarized data of mPAP and mRVP in different groups. Data are expressed as the mean \pm SEM. $n=7 /$ group. ${ }^{\circ} P<0.01$ vs control. ${ }^{\mathrm{f}} \mathrm{P}<0.01$ vs vehicle. mPAP, mean pulmonary artery pressure; mRVP, mean right ventricle pressure; RV, right ventricle; $\mathrm{PAH}$, pulmonary artery hypertension.

\section{Discussion}

The major finding in this study is that the intravenous injection of MSC-MVs or MSCs could similarly decrease pulmonary pressure and RV pressure and reduce RV hypertrophy and pulmonary arteriole remodeling during the development of PAH in rats.

$\mathrm{PAH}$ is a life-threatening disease resulting from a variety of heart and lung diseases. The main pathological changes in PAH include persistent pulmonary artery hypertension, pulmonary vascular intima thickening, severe hypoxia, and eventually right heart remodeling and failure ${ }^{[17]}$. Clinically, $\mathrm{PAH}$ is refractory to most conventional pharmacological therapies. Stem cell transplantation therapy has been shown to improve the vascular endothelial function of the pulmonary $\operatorname{arterie}^{[3,13,18]}$, and their therapeutic potential for PAH has been recognized in several studies ${ }^{[4,5,19,20]}$. Bone marrow-derived MSCs could improve RV function and decrease pulmonary arteriolar wall thickness and area in MCT-induced PAH rats ${ }^{[4]}$. Two other groups have shown that the intratracheal administration of MSCs could attenuate pulmonary artery hypertension and pulmonary vascular resistance in PAH mice ${ }^{[5,21]}$. In the early stage (1 week after MCT administration) of PAH development, MSC treatment has been demonstrated to attenuate PAP and pulmonary remodeling, which are accompanied by the inhibition of the transforming growth factor $\beta$ (TGF $\beta$ )/ Smad2 signaling pathway ${ }^{[2]}$. In addition, because transplanted MSCs were found in the lung parenchyma instead of the walls of pulmonary vessels, the paracrine effects of MSCs should be responsible for the role of MSCs in pulmonary remodeling. Indeed, the paracrine mechanism has been proposed to be one of the underlying mechanisms that contributes to the therapeutic effect of transplanted stem cells ${ }^{[7,8,22]}$.

MVs are small heterogeneous extracellular vesicles that have been isolated from diverse cell types, including platelets, dendritic cells, endothelial cells, and MSCs. The proteins and genetic materials (mRNAs and microRNAs) carried by MVs could be transferred to target neighboring and distant cells. Accumulating evidence has indicated that MVs could modulate various processes, such as vascular function, angiogenesis, cell proliferation, inflammation, and coagulation ${ }^{[10,23,24]}$. Cord blood-derived MSC-MVs may promote angiogenesis in vitro and in vivo ${ }^{[25]}$. A recent study showed that the administration of MSC-exosomes can promote functional recovery and neurovascular plasticity in a stroke mode ${ }^{[10]}$. Later on, this group reported that the protective mechanism of MSC-exosomes is through transferring microRNA-133b to astrocytes and neurons ${ }^{[26]}$. Additionally, MSC-MVs have been shown to protect kidneys against injury via stimulating proliferation and 
A

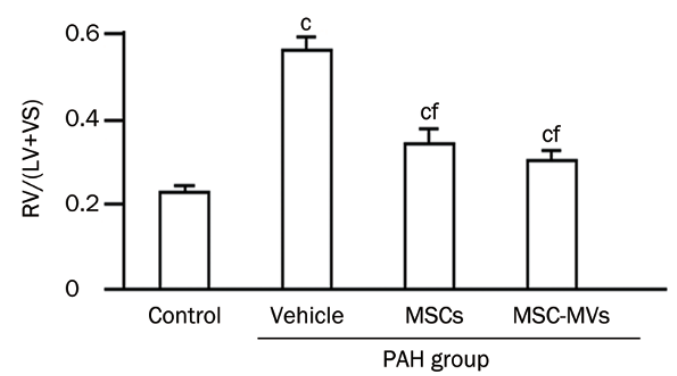

B
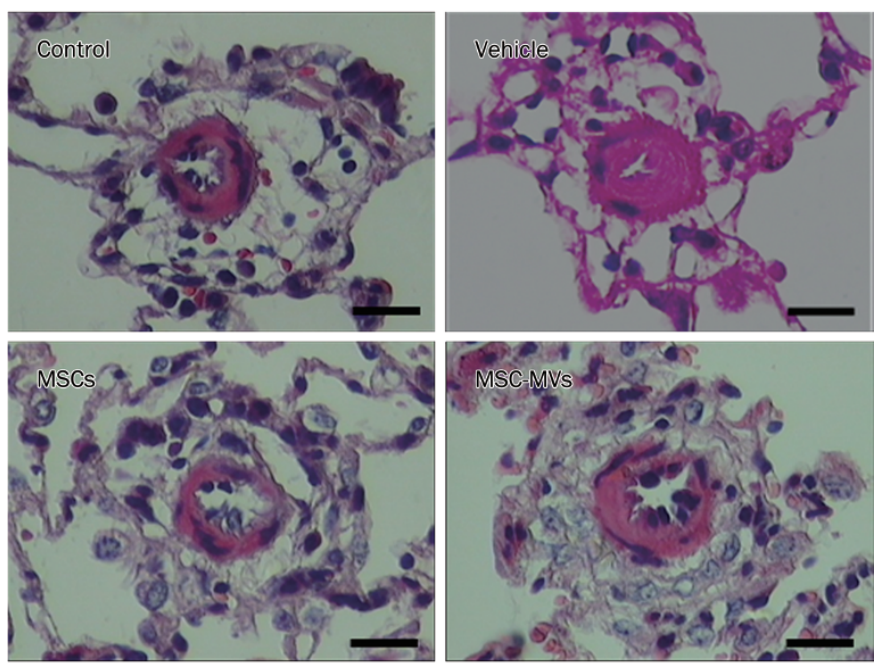

C

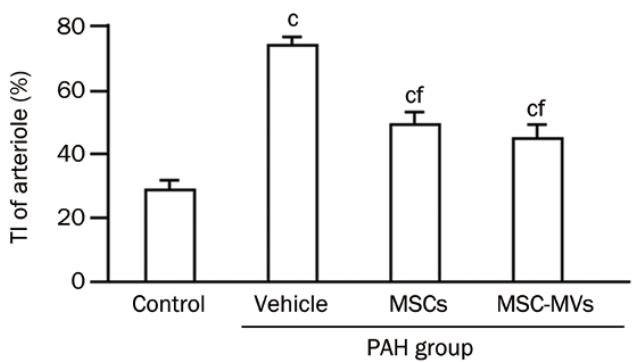

D

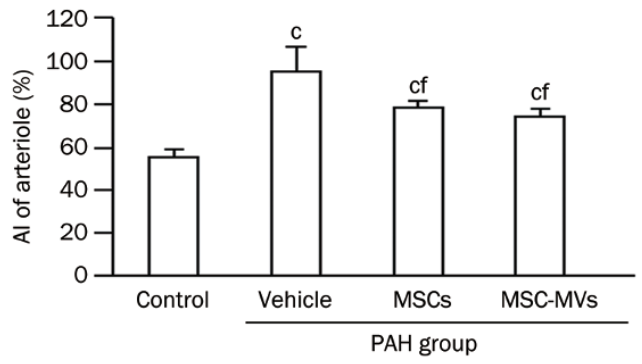

Figure 4. MSC-MVs and MSCs alleviate RV hypertrophy and pulmonary artery remodeling in $\mathrm{PAH}$ rats. (A) Weight ratio of $\mathrm{RV}$ to left ventricle (LV)+ventricle septum (VS) in control, vehicle, MSC, and MSC-MV groups. (B) Representative images of the pulmonary arteriole in different groups. Scale bar: $50 \mu \mathrm{m}$. (C and D) Summarized data of the pulmonary arteriole $\mathrm{TI}$ and $\mathrm{Al}$ in different groups. Data are expressed as the mean \pm SEM. $n=7$ /group. ${ }^{\mathrm{C}} P<0.01$ vs control; ${ }^{\mathrm{f}} P<0.01$ vs vehicle. $\mathrm{PAH}$, pulmonary artery hypertension; $\mathrm{Tl}$, thickness index; $\mathrm{Al}$, area index. decreasing the apoptosis of tubular epithelial cells ${ }^{[7-9]}$.

In the present study, the isolated MSC-MVs positively stained with annexin V and the MSC specific marker CD29 and displayed MV general spherical characteristics under TEM. According to the latest report from the International Society for Extracellular Vesicles (ISEV), MVs and exosomes are two major types of extracellular vesicles that can be differentiated by their size and content ${ }^{[27]}$. Exosomes are defined as extracellular vesicles ranging from 40 to $100 \mathrm{~nm}$ in size, and MVs are those with a larger size $(100-1000 \mathrm{~nm})^{[28]}$. However, Taylor's group found that $100-180-\mathrm{nm}$ vesicles exhibit CD63, a typical marker of exosomes, as determined by NTA ${ }^{[29]}$. In Lee's study, the "exosomes" were isolated through low-speed centrifugations followed by filtering the culture medium through a $0.2-\mu \mathrm{m}$ filter and then pelleting by ultracentrifugation $^{[12]}$. In our study, we isolated the vesicles by the same procedures, except for filtration through a filter. The NTA results showed that the size of these MSC-MVs mainly (>95\%) ranged from 40 to $300 \mathrm{~nm}$. To investigate the possible effects of MSCMVs on treating PAH, we systemically administered MSCMVs to an MCT-induced PAH rat model. Our data revealed that MSC-MV treatment attenuated $\mathrm{mPAP}$ and $\mathrm{mRVP}$ in PAH rats compared to the vehicle group, with similar effects on the MSC treatment group. We also observed that MSC-MV treatment reduced RV hypertrophy. Furthermore, pulmonary arteriole wall thickness and area were decreased, as revealed by H\&E staining of the lung tissue in the MSC-MV group. These data are in agreement with a previous study showing that the intravenous injection of MSCs can improve RV function in MCT-induced PAH mice ${ }^{[4]}$ and are also supported by Lee's findings in a hypoxia-induced PAH mouse model ${ }^{[12]}$.

MicroRNAs are small endogenous non-coding RNAs that regulate a wide range of biological processes, including cell apoptosis, differentiation, and cell proliferation. Extracellular vesicles are the main vehicles for circulating microRNAs and have been considered to be a novel mechanism of cellto-cell communication ${ }^{[30]}$. Recent studies have shown that microRNAs carried by exosomes play a critical role in treating stroke ${ }^{[26]}$ and hypoxia-induced $\mathrm{PAH}^{[12]}$ via regulating the gene expression of recipient cells. Based on these observations, we speculate that MSC-MV-carried microRNAs could contribute to the protective effect of MSC-MVs in our study. Although the precise mechanism of MSC-MV-mediated effects needs further investigation, our work provides an explanation for the observed effects of MSCs. Of note, there could be several benefits of using MVs versus cells. First, MVs should easily pass through the capillary network because they are nanoscale vesicles. Second, the potential risk of cell transplantation could be avoided.

In conclusion, our data demonstrate that MSC-MV treatment could attenuate mPAP and mRVP, and reduce RV hypertrophy and pulmonary remodeling in a MCT-induced PAH rat model. These data suggest that MSC-MVs could serve as a novel cell-free therapeutic source for treating PAH. 


\section{Acknowledgements}

This work was supported by the National Heart, Lung, and Blood Institute (HL-098637 to Yan-fang CHEN), National Natural Science Foundation of China (81370242 and 81270195), Guangdong Natural Science Foundation (S2013010015005), Science and Technology Planning Project of Guangdong Province (2012B031800224 and 2011B031800249), and Science and Technology Innovation Fund of Guangdong Medical College (STIF201128).

\section{Author contribution}

Jian-ying CHEN and Yan-fang CHEN designed the study; Ran AN, Zhen-jun LIU, Jing-hu LIU, Mian-ming HONG, and Meng-yuan XIAO conducted the experiment; Jian-ying CHEN, Ran AN, Jin-ju WANG, and Shu-zhen CHEN analyzed the data; and Jian-ying CHEN, Jin-ju WANG, Shu-zhen CHEN, and Yan-fang CHEN wrote the paper.

\section{References}

1 Farber HW, Loscalzo J. Pulmonary arterial hypertension. N Engl J Med 2004; 351; 1655-65.

2 Archer SL, Weir EK, Wilkins MR. Basic science of pulmonary arterial hypertension for clinicians: new concepts and experimental therapies. Circulation 2010; 121: 2045-66.

3 Zhang ZH, Lu Y, Luan Y, Zhao JJ. Effect of bone marrow mesenchymal stem cells on experimental pulmonary arterial hypertension. Exp Ther Med 2012; 4: 839-43.

4 Umar S, de Visser YP, Steendijk P, Schutte Cl, Laghmani el H, Wagenaar GT, et al. Allogenic stem cell therapy improves right ventricular function by improving lung pathology in rats with pulmonary hypertension. Am J Physiol Heart Circ Physiol 2009; 297: H1606-16.

5 Baber SR, Deng W, Master RG, Bunnell BA, Taylor BK, Murthy SN, et al. Intratracheal mesenchymal stem cell administration attenuates monocrotaline-induced pulmonary hypertension and endothelial dysfunction. Am J Physiol Heart Circ Physiol 2007; 292: H1120-8.

6 Ratajczak J, Wysoczynski M, Hayek F, Janowska-Wieczorek A, Ratajczak MZ. Membrane-derived microvesicles: important and underappreciated mediators of cell-to-cell communication. Leukemia 2006; 20: 1487-95.

7 He J, Wang Y, Sun S, Yu M, Wang C, Pei X, et al. Bone marrow stem cells-derived microvesicles protect against renal injury in the mouse remnant kidney model. Nephrology (Carlton) 2012; 17: 493-500.

8 Bruno S, Grange C, Deregibus MC, Calogero RA, Saviozzi S, Collino F, et al. Mesenchymal stem cell-derived microvesicles protect against acute tubular injury. J Am Soc Nephrol 2009; 20: 1053-67.

9 Gatti S, Bruno S, Deregibus MC, Sordi A, Cantaluppi V, Tetta C, et al. Microvesicles derived from human adult mesenchymal stem cells protect against ischaemia-reperfusion-induced acute and chronic kidney injury. Nephrol Dial Transplant 2011; 26: 1474-83.

10 Xin H, Li Y, Cui Y, Yang JJ, Zhang ZG, Chopp M. Systemic administration of exosomes released from mesenchymal stromal cells promote functional recovery and neurovascular plasticity after stroke in rats. J Cereb Blood Flow Metab 2013; 33: 1711-5.

11 Wang J, Chen S, Ma X, Cheng C, Xiao X, Chen J, et al. Effects of endothelial progenitor cell-derived microvesicles on hypoxia/ reoxygenation-induced endothelial dysfunction and apoptosis. Oxid Med Cell Longev 2013; 2013: 572729.

12 Lee C, Mitsialis SA, Aslam M, Vitali SH, Vergadi E, Konstantinou G, et al. Exosomes mediate the cytoprotective action of mesenchymal stromal cells on hypoxia-induced pulmonary hypertension. Circulation 2012; 126: 2601-11.

13 Kanki-Horimoto S, Horimoto H, Mieno S, Kishida K, Watanabe F, Furuya $\mathrm{E}$, et al. Implantation of mesenchymal stem cells overexpressing endothelial nitric oxide synthase improves right ventricular impairments caused by pulmonary hypertension. Circulation 2006; 114: I181-5.

14 de Jong OG, Verhaar MC, Chen Y, Vader P, Gremmels H, Posthuma G, et al. Cellular stress conditions are reflected in the protein and RNA content of endothelial cell-derived exosomes. J Extracell Vesicles 2012. doi: 10.3402/jev.v1i0.18396.

15 Chen J, Chen J, Chen S, Zhang C, Zhang L, Xiao X, et al. Transfusion of CXCR4-primed endothelial progenitor cells reduces cerebral ischemic damage and promotes repair in $d b / d b$ diabetic mice. PLoS One 2012; 7: e50105.

16 Kimura S, Egashira K, Chen L, Nakano K, Iwata E, Miyagawa M, et al. Nanoparticle-mediated delivery of nuclear factor kappaB decoy into lungs ameliorates monocrotaline-induced pulmonary arterial hypertension. Hypertension 2009; 53: 877-83.

17 Bull TM. New and future therapies in pulmonary arterial hypertension. Semin Respir Crit Care Med 2005; 26: 429-36.

18 Raoul W, Wagner-Ballon O, Saber G, Hulin A, Marcos E, Giraudier S, et al. Effects of bone marrow-derived cells on monocrotaline- and hypoxia-induced pulmonary hypertension in mice. Respir Res 2007; 8: 8.

19 Weiss DJ, Bertoncello I, Borok Z, Kim C, Panoskaltsis-Mortari A, Reynolds S, et al. Stem cells and cell therapies in lung biology and lung diseases. Proc Am Thorac Soc 2011; 8: 223-72.

20 Zhao YD, Courtman DW, Deng Y, Kugathasan L, Zhang Q, Stewart DJ. Rescue of monocrotaline-induced pulmonary arterial hypertension using bone marrow-derived endothelial-like progenitor cells: efficacy of combined cell and eNOS gene therapy in established disease. Circ Res 2005; 96: 442-50.

21 Xie J, Hu D, Niu L, Qu S, Wang S, Liu S. Mesenchymal stem cells attenuate vascular remodeling in monocrotaline-induced pulmonary hypertension rats. J Huazhong Univ Sci Technolog Med Sci 2012; 32: 810-7.

22 Collino F, Deregibus MC, Bruno S, Sterpone L, Aghemo G, Viltono L, et al. Microvesicles derived from adult human bone marrow and tissue specific mesenchymal stem cells shuttle selected pattern of miRNAs. PLoS One 2010; 5: e11803.

23 Sinauridze El, Kireev DA, Popenko NY, Pichugin AV, Panteleev MA, Krymskaya OV, et al. Platelet microparticle membranes have 50- to 100-fold higher specific procoagulant activity than activated platelets. Thromb Haemost 2007; 97: 425-34.

24 Distler JH, Pisetsky DS, Huber LC, Kalden JR, Gay S, Distler O. Microparticles as regulators of inflammation: novel players of cellular crosstalk in the rheumatic diseases. Arthritis Rheum 2005; 52 : 3337-48.

25 Zhang HC, Liu XB, Huang S, Bi XY, Wang HX, Xie LX, et al. Microvesicles derived from human umbilical cord mesenchymal stem cells stimulated by hypoxia promote angiogenesis both in vitro and in vivo. Stem Cells Dev 2012; 21: 3289-97.

26 Xin H, Li Y, Liu Z, Wang X, Shang X, Cui Y, et al. MiR-133b promotes neural plasticity and functional recovery after treatment of stroke with multipotent mesenchymal stromal cells in rats via transfer of exosome-enriched extracellular particles. Stem Cells 2013; 31: 2737-46.

27 Hill AF, Pegtel DM, Lambertz U, Leonardi T, O'Driscoll L, Pluchino $\mathrm{S}$, et al. ISEV position paper: extracellular vesicle RNA analysis 
and bioinformatics. J Extracell Vesicles 2013. doi: 10.3402/jev. v2i0.22859.

28 Mathivanan S, Ji H, Simpson RJ. Exosomes: extracellular organelles important in intercellular communication. J Proteomics 2010; 73 : 1907-20.

29 Gercel-Taylor C, Atay S, Tullis RH, Kesimer M, Taylor DD. Nanoparticle analysis of circulating cell-derived vesicles in ovarian cancer patients. Anal Biochem 2012; 428: 44-53.

30 Valadi H, Ekström K, Bossios A, Sjöstrand M, Lee JJ, Lötvall JO. Exosome-mediated transfer of mRNAs and microRNAs is a novel mechanism of genetic exchange between cells. Nat Cell Biol 2007; 9: $654-9$. 